\title{
Balanced Sequences and Optimal Routing
}

\author{
Eitan ALTMAN* $\quad$ Bruno GAUJAL ${ }^{\dagger} \quad$ Arie HORDIJK \\ September, 1997
}

\begin{abstract}
The objective pursued in this paper is two-fold. The first part gives an overview of the following combinatorial problem: is it possible to construct an infinite sequence over $n$ letters where each letter is distributed as "evenly" as possible and appears with a fixed rate? The second objective of the paper is to relate this construction to the framework of optimal routing in queueing networks. We show under rather general assumptions that the optimal deterministic routing in stochastic event graphs is such a sequence.
\end{abstract}

Keywords Balanced sequences, multimodularity, stochastic event graphs, optimal control.

\section{Introduction}

It is a rather general problem to consider a system with multiple resources and tasks. Tasks can be performed by any resource and arrive in the system sequentially. The problem is to construct a routing of the tasks to the resources to minimize a given cost function. Such models are common in multiprocessor systems and communication networks, where the cost function may be the combined load in the resources.

Here, we show that under rather general assumptions, the optimal routing sequence in terms of expected average workload in each resource is given by a balanced sequence, that is a sequence in which the option to route towards a given resource, is taken in an evenly distributed fashion.

${ }^{*}$ INRIA, BP 93, 2004 Route des Lucioles, 06902 Sophia Antipolis Cedex, France. E-mail: altman@sophia.inria.fr. URL:http://www.inria.fr:80/mistral/personnel/Eitan.Altman/me.html

${ }^{\dagger}$ INRIA/CNRS/INRIA, BP 93, 2004 Route des Lucioles, 06902 Sophia Antipolis Cedex, France. E-mail: gaujal@sophia.inria.fr.

${ }^{\ddagger}$ Dept. of Mathematics and Computer Science, Leiden University, P.O.Box 9512, 2300RA Leiden, The Netherlands. E-mail: hordijk@wi.leidenuniv.nl. The research of Arie Hordijk was done while he was on sabbatical leave at INRIA, Sophia-Antipolis; it has been partially supported by the Ministère Français de l'Éducation Nationale et de l'Enseignement Supérieur et de la Recherche. 
This motivates the first part of the paper which is essentially based on word combinatorics and uses its specific vocabulary. This part relies heavily on results in [9, 17]. The problem of balanced sequences and exactly covering sequences has been studied using combinatorial as well as arithmetic techniques [20, 23, 18, 7, 16].

However, in these studies, the analysis of balanced sequences was done per se and did not present any motivations or applications. In particular, the use of these constructions in discrete event dynamic systems may not have been discovered before the seminal work of Hajek, [10] in 1985. This work solves the one dimensional case problem, further generalized in [1]. The goal of the present paper is to extend the same type of results to a multidimensional case, which is surprisingly more difficult and of different nature as the one dimensional case.

This is done in the second part of this paper, where we will show an application of balanced sequences for routing problems. This part uses results from convex analysis, mainly from $[10,2,1]$. The main results that are used here are of two different kinds. First, we use the fact that the workload as well as the waiting time of customers entering a $(\max ,+)$ linear system are multimodular functions, under fairly general assumption ( stationarity of the arrival process and of the service times)([1]). Then, we use general theorems from [2] that prove that multimodular functions are minimized by regular sequences. The superposition of several such sequences being a balanced sequence, this is the basis of the main result of this paper.

It is interesting to exhibit this link between balanced sequences and scheduling problems, such as routing among several systems.

More precisely, the paper is structured as follows. In the second section, we introduce a formal definition of balanced sequence and we present an overview on their properties. The section 3 makes the link between the notion of balanced sequences and the optimal scheduling in networks and is also devoted to prove the optimality of balanced sequences for routing customers is a multiple queue system. Section 4 presents special cases for which the optimal rates can be computed.

\section{Balanced sequences}

In this section, we will present the notion of balanced sequences, which is closely related to the notion of Sturmian sequences [14] as well as exactly covering sequences. This presentation is not exhaustive and many other related articles can be consulted for further investigation on this topic $[22,11,3,5,20,7]$. Although the section is self-contained and presents several result which are of interest by their own, we mostly focus the rate problem (see Problem 2), which will be used in the application section $(\S 3)$.

\subsection{Preliminaries}

Let $\mathcal{A}$ be a finite alphabet and $\mathcal{A}^{\mathbb{Z}}$ the set of sequences defined on $\mathcal{A}$. 
If $u \in \mathcal{A}^{\mathbb{Z}}$, then a word $W$ of $u$ is a finite subsequence of consecutive letters in $u$ : $W=u_{a} u_{a+1} \cdots u_{a+k-1}$. The integer $k$ is the length of $W$ and will be denoted $|W|$.

If $a \in \mathcal{A},|W|_{a}$ is the number of $a$ 's in the word $W$.

Definition 2.1. The sequence $u \in \mathcal{A}^{\mathbb{Z}}$ is balanced if for any two words $W$ and $W^{\prime}$ in $u$ of same length, and any $a \in \mathcal{A}$,

$$
-1 \leqslant|W|_{a}-\left|W^{\prime}\right|_{a} \leqslant 1
$$

If $a \in \mathcal{A}$, we also define the indicator in $u$ of the letter $a$ as the function $\delta_{a}(u): \mathcal{A}^{\mathbb{Z}} \rightarrow$ $\{0,1\}^{\mathbb{Z}}$ by, $\delta_{a}(u)_{i}=1$ if $u(i)=a$ and 0 otherwise. The support in $u$ of the letter $a$ is the set $S_{a}=\left\{i \in \mathbb{Z}: \delta_{a}(u)_{i}=1\right\}$.

For any real number $x,\lfloor x\rfloor$ will denote the largest integer smaller or equal to $x$ and $\lceil x\rceil$ will denote that smallest integer larger or equal to $x$.

Lemma 2.2. If a sequence $u \in \mathcal{A}^{\mathbb{Z}}$ is balanced, then for any a $\in \mathcal{A}$, there exists a real number $p, 0 \leqslant p \leqslant 1$ such that

$$
\lim _{n \rightarrow \infty} \frac{1}{n} \sum_{i=0}^{n} \delta_{a}(u)_{i}=\lim _{n \rightarrow-\infty} \frac{1}{n} \sum_{i=n}^{0} \delta_{a}(u)_{i}=p_{a} .
$$

$p_{a}$ is called the rate of $\delta_{a}(u)$.

Proof. Let us define $s_{n}=\sum_{i=0}^{n} \delta_{a}(u)_{i}$ and remark that $s_{n}+s_{m}-1 \leqslant s_{n+m} \leqslant s_{n}+s_{m}+1$. The rest of the proof is classical by sub-additivity arguments. The proof for $\left\{\delta_{a}(u)_{n}\right\}_{n \leqslant 0}$ is similar. The fact that both limits coincide is obvious.

Note that the sum of the rates for all letters in a sequence $u$ is one.

$$
\sum_{a \in \mathcal{A}} p_{a}=1
$$

Now, we can present the main result which founds the theory of balanced sequences.

Theorem 2.3 (Morse and Hedlund). Suppose a sequence $d \in\{0,1\}^{\mathbb{Z}}$ is balanced with asymptotic rate $p=1 / \alpha$ for letter 1 . The support $S_{1}$ of $d$ satisfies one of the following cases.

(a) (irrational case) $p$ is irrational and that exists $\phi \in \mathbb{R}$ such that

$$
S_{1}=\{\lfloor i \alpha+\phi\rfloor\}_{i \in \mathbb{Z}} \quad \text { or } \quad S_{1}=\{\lceil i \alpha+\phi\rceil\}_{i \in \mathbb{Z}}
$$

(b) (periodic case) $p \in \mathbb{Q}$ and there exists $\phi \in \mathbb{Q}$ such that

$$
S_{1}=\{\lfloor i \alpha+\phi\rfloor\}_{i \in \mathbb{Z}}
$$


(c) (skew case) $p \in \mathbb{Q}(p=k / n)$ and there exists $m \in \mathbb{Z}$ such that

$$
S_{1}=\{\lfloor i n / k+m\rfloor\}_{i<k} \cup\{\lfloor i n / k-1 / k+m\rfloor\}_{i>0}
$$

or

$$
S_{1}=\{\lfloor i n / k+m\rfloor\}_{i>0} \cup\{\lfloor i n / k-1 / k+m\rfloor\}_{i<k}
$$

Sequences for which the support is of the form $S_{1}=\{\lfloor i \alpha+\phi\rfloor\}_{i \in \mathbb{Z}}$ are called bracket sequences. This theorem shows the relation that exists between balanced sequences and bracket sequences. The irrational case is the easiest case and can be characterized (see Theorem 2.17). The two rational cases are more difficult to study. Our main objective for studying balanced sequences is their application in routing control (see sections 3 and 4). In our analysis, only the right tail of a sequence, that is $\left\{u_{i}\right\}_{i \geqslant i_{0}}$ for some $i_{0}$ in $\mathbb{N}$ maters. Also, a sequence $u$ and a shifted version, $u^{\prime}$ will have the same asymptotic performance (see equation (7)). Hence, we consider such two sequences as being equivalent. Note that this allows us to consider that the skew case and the rational case are equivalent.

Definition 2.4. A sequence $d \in\{0,1\}^{\mathbb{Z}}$ is (ultimately) regular if there exists two real numbers $\theta$ and $p$ (and an integer $k$ ) such that for all $(n \geqslant k) n, d_{n}=\lfloor(n+1) p+\theta\rfloor-\lfloor n p+\theta\rfloor$.

Note that $d_{n}=\lfloor(n+1) p+\theta\rfloor-\lfloor n p+\theta\rfloor$ is equivalent to $S_{1}=\left\{\left\lceil n \frac{1}{p}+\phi\right\rceil\right\}_{n \in \mathbb{Z}}$ with $\phi=-1-\theta / p$.

Theorem 2.5. Let $u \in \mathcal{A}^{\mathbb{Z}}$.

(i)- If $\delta_{a}(u)$ is regular for all $a \in \mathcal{A}$, then $u$ is balanced.

(ii)- If $u$ is balanced, then $\delta_{a}(u)$ is ultimately regular for all $a \in \mathcal{A}$.

Proof. (i) is straightforward.

(ii) is a direct consequence of Theorem 2.3, since in all three cases, the sequence $\delta_{a}(u)$ is ultimately regular. An elementary proof of (ii) which does not use Theorem 2.3 can be found in [21].

In [13], the sequence $u$ is said to have the reduction property if $\delta_{u}(a)$ is regular for all $a \in \mathcal{A}$. Corollary 2.5 shows that $u$ has the reduction property (ultimately) if and only if it is balanced.

\subsection{Constant gap sequences}

Constant gap sequences are strongly balanced sequences, in the following sense.

Definition 2.6. A sequence $G$ is constant gap if for any letter $a, \delta_{a}(G)$ is periodic, with a period of the form $0 \cdots 010 \cdots 0$.

Note that this explains the fact that $G$ is said to have constant gaps for the letter $a$, since each $a$ is separated from the next $a$ in $G$ by a constant number of letters. 
Proposition 2.7. Constant gap sequences are balanced.

Proof. For each letter $a, \delta_{a}(G)$ is of the form $\left(0^{n} 10^{m}\right)^{\omega}$. Therefore, $\delta_{a}(G)$ is regular with $p=1 /(m+n+1)$ and $\theta=m /(m+n+1)$. Using the characterization of balanced sequences given in Theorem 2.5, this shows that $G$ is balanced.

Proposition 2.8. Constant gap sequences are periodic.

Proof. For each letter $a, \delta_{a}(G)$ is periodic with period $p_{a}$. The period of $G$ is $l c m\left(p_{a}, a \in\right.$ $\mathcal{A})$.

In the next lemma, we give a characterization of constant gap sequences that stresses the fact that constant gap is some kind of strong balance.

Proposition 2.9. $G$ is constant gap if and only if, for any two finite words, $W$ and $W^{\prime}$ included in $G$ with ||$W|-| W^{\prime}|| \leqslant 1$, then for each letter $a,\left.|| W\right|_{a}-\left|W^{\prime}\right|_{a} \mid \leqslant 1$.

Proof. Let $a$ be a letter in the alphabet.

First, assume that $G$ is constant gap. If $|W|_{a}-\left|W^{\prime}\right|_{a} \geqslant 2$, then, necessarily, $|W|-\left|W^{\prime}\right| \geqslant 2$. Conversely, let $W=a U a$ and $W^{\prime}=a U^{\prime} a$ be any two words in $G$ with no $a$ in the subwords $U$ and $U^{\prime}$. If $|U| \geqslant\left|U^{\prime}\right|+1$, then we have ||$U|-| W^{\prime}|| \leqslant 1$ and $\left.|| U\right|_{a}-\left|W^{\prime}\right|_{a} \mid=2$. This is a contradiction. Therefore $|U|=\left|U^{\prime}\right|$ and $G$ is constant gap.

Since a constant gap sequence is balanced, each letter appears with a given rate in the sequence. note however that since a constant gap sequence is necessarily periodic, the rate of each letter is rational.

As we will do in section 2.4 for the case of balanced sequences, we now address the following question:

Problem 1: Given a set $\left(p_{1}, \cdots, p_{N}\right)$, with $p_{1}+\cdots+p_{N}=1$ is it possible to construct a constant gap sequence on $N$ letters with rates $\left(p_{1}, \cdots, p_{N}\right)$ ?

We will not solve this problem for a general $N$. We will only give some properties of the set $\left(p_{1}, \cdots, p_{N}\right)$ which will be useful in the following. A non-effective characterization of such $\left(p_{1}, \cdots, p_{N}\right)$ is given in [23], under the name exact covering sequences.

Definition 2.10. The set of couples $\left\{\left(\theta_{i}, g_{i}\right), i=1 \cdots N\right\}$ is called an exact covering sequence if for every nonnegative integer $n$, there exists one and only one $1 \leqslant i \leqslant N$ such that $n=\theta_{i} \bmod g_{i}$.

As a general remark, note that $\left(p_{1}, \cdots, p_{N}\right)$ are rational numbers of the form $p_{i}=k_{i} / d$, with $d$ the smallest period of $G$. Therefore, we have, $\sum_{i} k_{i} / d=1$ and for each $i, k_{i}$ divides $d$. By definition of the rates, we also have $p_{a}=1 / q_{a}$ for all letters.

We have the following result.

Proposition 2.11. The rates $\left(p_{1}, \cdots, p_{N}\right)$ are constant gap if there exists $N$ numbers called phases, $\theta_{1}, \cdots \theta_{N}$ such that the couples $\left\{\left(\theta_{i}, q_{i}\right), i=1 \cdots N\right\}$ form an exact covering sequence. 
Proof. This property is a simple rewriting of the fact that each letter $a_{i}$ in a constant gap sequence appears every $\left\{\theta_{i}+k q_{i}, \quad k \in \mathbb{N}\right\}$.

Now, suppose that $\left\{\left(\theta_{i}, q_{i}\right), i=1 \cdots N\right\}$ is an exact covering sequence. Then in the series

$$
S(x) \stackrel{\text { def }}{=} \sum_{i=1}^{k} \sum_{k \geqslant 0} x^{\theta_{i}+k q_{i}},
$$

the coefficient of $x^{n}$ in this series is equal to $1, \forall n \geqslant 0$. Therefore, we have

$$
S(x)=\sum_{i=1}^{k} \frac{x_{i}^{\theta}}{1-x^{q_{i}}}=\frac{1}{1-x} .
$$

Using this characterization we have the following interesting property.

Lemma 2.12 ([18]). Assume $\left\{\left(\theta_{i}, q_{i}\right), i=1 \cdots N\right\}$ is an exact covering sequence and that $P=\max _{i} q_{i}$. Then $P$ appears at least twice in the set $q_{1}, \cdots, q_{N}$.

Proof. The proof given here is similar to the discussion in [23] on exact covering sequences. Let $w \stackrel{\text { def }}{=} e^{2 i \pi / r}$ for some integer $r>1$. By definition, $w$ is a primitive $r$-th root of one. We have:

$$
(w-x) S(x)=\sum_{i=1}^{k} \frac{(w-x) x_{i}^{\theta}}{1-x^{q_{i}}}=\frac{w-x}{1-x} .
$$

Let $x \rightarrow w$. This yields

$$
\sum_{i: r \mid q_{i}} \frac{-w^{\theta_{i}}}{-q_{i} w^{q_{i}-1}}=0 .
$$

Now, take $r=P$. The set $\left\{i: P \mid q_{i}\right\}$ is exactly the set $\left\{i: P=q_{i}\right\}$. Equation (1) specified for $r=P$ can be written

$$
\sum_{i: q_{i}=P} w^{\theta_{i}}=0
$$

This implies that the set $\left\{i: P=q_{i}\right\}$ cannot be reduced to a single point since $w$ is not zero.

To give some concrete examples, we consider the cases where $N$ is small. First, note that in the case where the $k_{i}$ are not all equal, (assume $k_{1}$ is the largest of all), we have

$$
\sum_{i \neq 1} k_{i} / k_{1}=d / k_{1}-1=l_{1},
$$

where $l_{1}$ is the gap between two letters $a_{1}$. This implies,

$$
l_{1} \leqslant N-2
$$


Proposition 2.13. There exists a constant gap sequence $G$ with rates $(p, 1-p)$ if and only if $p=1 / 2$.

Proof. Let $a$ be a letter in $G$ with gap $l$. Since the alphabet contains only two letters, $l=1$. This means $p=1 / 2$.

Proposition 2.14. There exists a constant gap sequence $G$ with rates $\left(p_{1}, p_{2}, p_{3}\right)$ if and only if the following holds: $\left(p_{1}, p_{2}, p_{3}\right)=(1 / 3,1 / 3,1 / 3)$ or $(1 / 2,1 / 4,1 / 4)$ (up to a permutation).

Proof. Assume that $\left(p_{1}, p_{2}, p_{3}\right) \neq(1 / 3,1 / 3,1 / 3)$ (otherwise, $G=(a b c)^{\omega}$ is constant gap). Using Equation (2), $l_{1}=1$ and $p_{1}=1 / 2$. Therefore, the sequence obtained from $G$ when removing all the letters $a_{1}$ is constant gap. Applying Lemma 2.13 shows that $p_{2}=p_{3}$. The only solution is $p_{1}=1 / 2, p_{2}=1 / 4$ and $p_{3}=1 / 4$. The associated constant gap sequence is $\left(a_{1} a_{2} a_{1} a_{3}\right)^{\omega}$.

Proposition 2.15. There exists a constant gap sequence $G$ with rates $\left(p_{1}, p_{2}, p_{3}, p_{4}\right)$ if and only if $\left\{p_{1}, p_{2}, p_{3}, p_{4}\right\}$ belongs to the set (up to a permutation),

$$
\{(1 / 4,1 / 4,1 / 4,1 / 4),(1 / 2,1 / 4,1 / 8,1 / 8),(1 / 2,1 / 6,1 / 6,1 / 6),(1 / 3,1 / 3,1 / 6,1 / 6)\} .
$$

Proof. We give a sketch of an elementary proof of this fact. If the rates are all equal, then $\left(p_{1}, p_{2}, p_{3}, p_{4}\right)=(1 / 4,1 / 4,1 / 4,1 / 4)$. Now, note that Equation 2 implies that if the rates are not all equal $l_{1} \leqslant 2$. We consider any letter $a_{i}, i \neq 1$, assume that the number of $a_{1}$ 's in between two $a_{i}$ 's is not constant and takes values $m$ and $n, m>n$. then we have $l_{i} \geqslant(n-1) l_{1}+n$ on one hand and $l_{i} \leqslant n\left(l_{1}\right)-3$ on the other hand. This is impossible since $l_{1} \leqslant 2$. Therefore, the number of $a_{1}$ 's in between two $a_{i}$ 's is constant. This is true for all $i$. The sequence formed by removing all $a_{1}$ 's is still constant gap. It has rates of the form $(1 / 3,1 / 3,1 / 3)$ or $(1 / 2,1 / 4,1 / 4)$. From this point a case analysis shows that the original sequence has rates $(1 / 2,1 / 4,1 / 8,1 / 8),(1 / 2,1 / 6,1 / 6,1 / 6) \operatorname{or}(1 / 3,1 / 3,1 / 6,1 / 6)\}$ by inserting the letter $a_{1}$ in a constant gap sequence over the letters $a_{2}, a_{3}, a_{4}$.

These few examples of constant gap sequences illustrate the fact that the rates in these sequences have very strong constraints.

\subsection{Characterization of balanced sequences}

Several studies have been recently done on balanced (or bracket) sequences $[13,20,21,16$, 15]. In [9], a characterization involving constant gap sequences is given.

Proposition 2.16 (Graham). Let $U$ be a balanced sequence on the alphabet $\{0,1\}$. Construct a new sequence $S$ by replacing in $U$, the subsequence of zeros by a constant gap sequence $G$ on an alphabet $\mathcal{A}_{1}$, and the subsequence of ones by a constant gap sequence $H$ on a disjoint alphabet $\mathcal{A}_{2}$. Then $S$ is balanced on the alphabet $\mathcal{A}_{1} \cup \mathcal{A}_{2}$. 
Proof. We give a proof similar to Hubert's proof ([11]). Let a be a letter in $\mathcal{A}_{1}$ (the proof is similar for a letter in $\mathcal{A}_{2}$. Let $W$ and $W^{\prime}$ be two words of $S$ of the same length. Then, the corresponding words $X$ and $X^{\prime}$ in $U$ verify $\left.|| W\right|_{0}-\left|W^{\prime}\right|_{0} \mid \leqslant 1$ since $U$ is balanced. If we keep only the 0 's in $X$ and $X^{\prime}$, then the corresponding $Z$ and $Z^{\prime}$ words in $G$ satisfy ||$Z|-| Z^{\prime}|| \leqslant 1$. Since $G$ is constant gap, and using Lemma 2.9, $\left.|| Z\right|_{a}-\left|Z^{\prime}\right|_{a} \mid \leqslant 1$. We end the proof noting that the construction of $Z$ and $Z^{\prime}$ implies $|Z|_{a}=|W|_{a}$ and $\left|Z^{\prime}\right|_{a}=\left|W^{\prime}\right|_{a}$.

Conversely, we have the following theorem.

Theorem 2.17 (Graham). Let $u \in \mathcal{A}^{\mathbb{Z}}$ be balanced and non periodic. Then there exists a partition of $\mathcal{A}$ into two sets $\mathcal{A}_{1}$ and $\mathcal{A}_{2}$ such that the sequence $v$ defined by:

$$
\begin{array}{ll}
v_{n}=1 & \text { if } \quad u_{n} \in \mathcal{A}_{1}, \\
v_{n}=0 & \text { if } \quad u_{n} \in \mathcal{A}_{2},
\end{array}
$$

is regular. Furthermore, the sequences $z_{1}$ and $z_{2}$ constructed from $u$ by keeping only the letters from $\mathcal{A}_{1}$ and $\mathcal{A}_{2}$ respectively have constant gaps.

The proof of this theorem was given by Graham [9] for bracket sequences. An independent later proof can be found in [11] for balanced sequences. The relation between balanced and bracket sequences given in Theorem 2.5 makes both proofs more or less equivalent.

\subsection{Rates in balanced sequences}

Now, also note that a balanced sequence has several asymptotic properties, such as the following lemma.

Let us formulate precisely the problem which we will study in this section.

Problem 2: Given a set $\left(p_{1}, \cdots, p_{N}\right)$, is it possible to construct a balanced sequence on $N$ letters with rates $\left(p_{1}, \cdots, p_{N}\right)$ ?

We will see in the following that this construction is not possible for all the values of the rates $\left(p_{1}, \cdots, p_{N}\right)$. If a n-tuple $\left(p_{1}, \cdots, p_{N}\right)$ makes the construction possible, such a tuple is said to be balanceable. A similar problem has been addressed in [9, 15, 7], Where relations between the rates in balanced sequences are studied.

\subsection{The case $N=2$}

This case is well known and balanced sequences with two letters have been extensively studied (see for example [6, 14]). The following result is known even if it is often given under different forms.

Theorem 2.18. For all $p, 0 \leqslant p \leqslant 1$, the set of rates $(p, 1-p)$ is balanceable. 
Proof. The proof is similar to the proof of the first part of Theorem 2.5. We construct a sequence $S$ as the support of the function $s(n)=\lfloor p n\rfloor-\lfloor p(n-1)\rfloor . S$ is a balanced sequence because the interval $] k, k+m\rfloor$ contains exactly $e=\lfloor p k+p m\rfloor-\lfloor p k\rfloor$ elements of $S$. Now, $\lfloor p m\rfloor+\lfloor p k\rfloor-\lfloor p k\rfloor \leqslant e \leqslant\lceil p m\rceil+\lfloor p k\rfloor-\lfloor p k\rfloor$. This shows the value of $e$ can differ by at most one when k varies so $S$ is a balanced sequence. If $S^{\prime}$ is the complementary set of $S$, then it should be clear that $S^{\prime}$ has asymptotic rate $1-p$ and $S^{\prime}$ is balanced because $S_{] k, k+m]}^{\prime}$ contains $m-e$ elements.

Note that the proof of Theorem 2.18 also gives a construction of a balanced sequence with the given rates.

\subsection{The case $N=3$}

The case $N=3$ is essentially different from the case $N=2$. In the case $N=2$, all possible rates are balanceable while when $N=3$, there is essentially only one set of rates which is balanceable. This result, when formulated under this form, was partly proved and conjectured in [13] and proved in [20]. In earlier papers by Morikawa, [16], a similar result is proved for bracket sequences. If Theorem 2.5 is used, then the result of Morikawa can be used directly to prove the following theorem. Therefore, we attribute this result to Morikawa, even if the result was stated differently.

Theorem 2.19 (Morikawa). A set of rates $\left(p_{1}, p_{2}, p_{3}\right)$ is balanceable if and only if,

$$
\left(p_{1}, p_{2}, p_{3}\right)=(4 / 7,2 / 7,1 / 7)
$$

or two rates are equal.

Proof. The proof of Morikawa is very technical since it does not use the balanced property for bracket sequences. If the balanced property is used, then the proof becomes very easy. We give a proof slightly simpler than the proof in [20]. First, assume that $p_{1}=p_{2}$. Then, let $S$ be a balanced sequence with two letters $\{a, b\}$ constructed with the rates $\left(p_{1}+p_{1}, p_{3}\right)$. In $S$, replace alternatively the " $a$ "s by the letters $a_{1}, a_{2}$, we get a sequence $S$ " on alphabet $\left\{a_{1}, a_{2}, b\right\}$ with rates $\left(p_{1}, p_{1}, p_{3}\right)$. Let us show that $S^{\prime}$ is balanced. Since $S$ is balanced, the number of " $a$ "s in an interval of length $m$ is $k$ or $k+1$, for some $k$. Now, for $S^{\prime}$, the number of " $a_{1}$ "s (resp. " $a_{2}$ "s) in such an interval is either $(k-1) / 2$ or $(k+1) / 2$ if $k$ is odd and $k / 2$ or $k / 2+1$ is $k$ is even. This proves that $S^{\prime}$ is balanced.

Now, assume that $\left(p_{1}, p_{2}, p_{3}\right)$ are three different numbers. We assume that $p_{1}>p_{2}>p_{3}$. We will try to construct a sequence $W$ with these respective rates on the alphabet $\{a, b, c\}$.

step 1: the sequence "aca" must appear in $W$.

There exists a pair of consecutive " $a$ " with no " $b$ " in between since $p_{1}>p_{2}$. This means that the sequences " $a a$ " or " $a c a$ " appear. If " $a a$ " appears, then a " $c$ " is necessarily surrounded by two "a"s.

step 2: the sequence "baab" must appear in $W$.

There exist a pair of consecutive " $b$ " with no " $c$ " in between. This sequence is of the form 
" $b a^{n} b$ ". Now, $n \leqslant 1$ is not possible because of the presence of "aca" and b-regularity. $n \geqslant 3$ implies the existence of " $a^{n-1} c a^{n-1}$ " by $a$-regularity which is incompatible with " $b a^{n} b$ " because of $b$-regularity. Therefore $n=2$. Note that this also implies the existence of " $a a$ " and of "abaaba".

step 3: the sequence "abacaba" appears in $W$.

the sequence $W$ must contain a " $c$ ". This " $c$ " is necessarily surrounded by two " $a$ "s since " $a a$ " exists by a-regularity. This group is necessarily surrounded by two " $b$ "s since "baab" exists, and consequently, necessarily surrounded by two " $a$ "s, since "abaaba" exists. We get the sequence "abacaba".

Last step: $W=(a b a c a b a)^{\omega}$.

No letter around this word can be a " $c$ " because "baab" exists. None can be a " $b$ " since "aca" exists. Therefore, they have to be two " $a$ "s. Then note that the following two letters cannot be a " $c$ " (because of the existence of "abaaba"), nor an " $a$ " (because of the existence of " $b a c$ ") so it is a " $b$ ", then followed necessarily by an " $a$ " (because " $a a$ " exists). At this point, we have the sequence " $\star a b a a b a c a b a a b a \star$ ". Both $\star$ s are necessarily " $c$ "s.

To end the proof, note that a "c" necessarily has the word "abaaba" on its right and the same word on its left. Finally note that the word "abaaba" is necessarily surrounded by two " $c$ "s. Therefore the sequence $W$ is periodic of the form $(a b a c a b a)^{\omega}$.

\subsection{The case $N=4$}

The case $N=4$ is very similar to the case $N=3$ for distinct rates. However when two rates are equal, this case is more complicated.

Theorem 2.20. A rate tuple $\left(p_{1}, p_{2}, p_{3}, p_{4}\right)$ with four distinct rates is balanceable if and only if $\left(p_{1}, p_{2}, p_{3}, p_{4}\right)=(8 / 15,4 / 15,2 / 15,1 / 15)$.

Proof. Again, it seems Morikawa has proved a similar result for bracket sequences. However, using the balanced property, the proof becomes much simpler. We suppose that $p_{1}>p_{2}>p_{3}>p_{4}$ and we show that there is only one balanced sequence with frequencies $p_{1}>p_{2}>p_{3}>p_{4}$ and those frequencies are $(8 / 15,4 / 15,2 / 15,1 / 15)$.

As a preliminary remark, note that if $p_{i}>p_{j}$, then there exists at least one word " $a_{i} \cdots a_{i}$ " that does not contain any $a_{j}$. This fact will be used several times in the following arguments.

The proof involves different steps.

Step 1: W contains the words "aca" or "ada" or "acda" or "adca".

There exists two consecutive " $a$ "s with no " $b$ " in between because $p_{1}>p_{2}$. Therefore, either "aa" or "aca" or "ada" or "acda" or "adca" exist. If "aa" exists, then, a "c" is surrounded by two " $a$ "s.

Step 2: W contains the word "baab"

First, we show that if a word " $b a^{n} b$ " exists, then $n=2$. Indeed, the fact that " $a d a$ " or " $a c a$ " or " $a c d a "$ or " $a d c a$ " exist makes " $b b$ " and "bab" impossible. On the other hand, if $n \geqslant 3$, the existence of " $a^{n-1} c a^{n-1}$ " is necessary by $a$-regularity and is incompatible with the existence of " $b a^{n} b$ " because of $b$-regularity. 
Now, if no word of the form " $b a^{n} b$ " exists, then there exist two consecutive " $b$ "s with one " $d$ " and no " $c$ " in between. This word $\left(s_{1}\right)$ is of the form: " $a^{i} b a^{j} d a^{k} b a^{l}$ ". Note that the numbers $i, j, k, l$ may be equal to zero but $i+j+k+l \geqslant 1$ by $b$-regularity.

There also exist two consecutive " $c$ "s with no " $d$ " in between. This word $\left(s_{2}\right)$, is of the form: " $c a^{m} b a^{n} c$ ". Note that $i, j, k, l, m, n$ are integers that can differ by at most one, the length of $s_{1},\left|s_{1}\right| \geqslant \max (4,2(n+m)+1) \geqslant n+m+3=\left|s_{2}\right|$. This is impossible by $c$-regularity.

step 3: the word "abacabaabacaba" exists in $W$. There exists two consecutive "c"s with no " $d$ " in between. From step 3 in the proof of Lemma 2.19, we know that a " $c$ " is necessarily surrounded by the word " $a b a$ ". Moreover, from step 4 in the proof of Lemma 2.19, we have: "abacabaabaUcaba", where $U$ is a word that contains no " $d$ " and no "c". $U$ cannot start with an " $a$ " (because of "bacab") cannot start with a " $b$ " (because of "aca") cannot start with a " $c$ " and cannot start with a " $d$ " by construction. Therefore $U$ has to be empty.

Step 4: $W$ is uniquely defined and is periodic of period "abacabadabacaba".

Somewhere, $W$ contains a " $d$ ". From this point on, we can extend the word uniquely as: "abacabadabacaba" around this " $d$ ", and the word "abacabaabacaba" has to be surrounded by two "d"s. This ends the proof.

To complete the picture, it is not difficult to see that,

Proposition 2.21. if the tuple $\left(p_{1}, p_{2}, p_{3}, p_{4}\right)$ is made of less than two distinct numbers, then it is balanceable.

Proof. First, if the rates are all equal, they are obviously balanceable. If three of them are equal, say $p_{1}=p_{2}=p_{3}$, then, we can construct a balanced sequence with rates $\left(3 p_{1}, p_{4}\right)$ and we construct a balanced sequence with rates $\left(p_{1}, p_{1}, p_{1}, p_{4}\right)$ by using Proposition 2.16 (where we take $G$ the constant gap sequence $\left(a_{1} a_{2} a_{3}\right)^{\omega}$ and $\left.H \stackrel{\text { def }}{=}\left(a_{4}\right)^{\omega}\right)$. If two pairs of rates are equal, say $p_{1}=p_{2}$ and $p_{3}=p_{4}$, then we construct a balanced sequence with rates $\left(2 p_{1}, 2 p_{3}\right)$ and we apply Proposition 2.16 .

If the tuple $\left(p_{1}, p_{2}, p_{3}, p_{4}\right)$ is made of exactly three distinct numbers, then this is a more complex case which is not studied here.

\subsection{The general case}

In this section, we are interested in the case of arbitrary $N$. First, note that Proposition 2.21 easily generalizes to any dimension.

Proposition 2.22. If the tuple $\left(p_{1}, p_{2}, p_{3}, \cdots, p_{N}\right)$ is made of less than two distinct numbers, then it is balanceable.

Proof. The proof is similar to the proof of Proposition 2.21. 
Proposition 2.23. If $\left(p_{1}, p_{2}, \cdots, p_{N}\right)$ is balanceable, then $(\underbrace{p_{1} / k, \cdots, p_{1} / k}_{k}, p_{2}, \cdots, p_{N})$ is balanceable.

Proof. The proof is very similar to that of Proposition 2.16. If $W$ is a balanced sequence with letters $\left\{a_{1}, \cdots, a_{N}\right\}$, consider the sequence $W^{\prime}$ constructed starting from $W$ and replacing each " $a_{1}$ " by an element of $\left(b_{1} ", " b_{2} ", \cdots, " b_{k}\right)$ in a cyclic way. Note that $W^{\prime}$ has the following set of rates, $\left(p_{1} / k, \cdots, p_{1} / k, p_{2}, \cdots, p_{N}\right)$.

Next, we show that $W^{\prime}$ is balanced. Since $W$ is balanced, for an arbitrary integer $m$, the number of " $a_{1}$ "s in an interval of length $m$ is $n$ or $n+1$, for some $n$. Now, for $W^{\prime}$, the number of " $b_{i}$ "s in such an interval is either $\lfloor(n-1) / k\rfloor$ or $\lfloor(n+1) / k\rfloor$. This proves that $W^{\prime}$ is balanced.

For the general case and distinct rates, it is natural to give the following conjecture (due to Fraenkel for bracket sequences):

Conjecture 2.24. A set of distinct rates $\left\{p_{1}, \cdots, p_{N}\right\}$ is balanceable if and only if

$$
\left\{p_{1}, \cdots, p_{N}\right\}=\left\{2^{N-1} /\left(2^{N}-1\right), \cdots, 2^{N-i} /\left(2^{N}-1\right), \cdots, 1 /\left(2^{N}-1\right)\right\} .
$$

We have not been able to prove this fact. Morikawa has also given some insight in this problem. It is not clear whether it has been completely proven. Here, we only have partial results given in the following lemmas.

Lemma 2.25. The rates $\left(2^{N-1} /\left(2^{N}-1\right), \cdots, 2^{N-i} /\left(2^{N}-1\right), \cdots, 1 /\left(2^{N}-1\right)\right)$ are balanceable, for all $N \in \mathbb{N}$.

This lemma is the "if" direction of the conjecture.

Proof. We construct a balanced sequence $W_{N}$ in the following inductive way. $V_{1}=a_{1}$, $V_{N}=V_{N-1} a_{N} V_{N-1}$ and $W_{N}=\left(V_{N}\right)^{\omega}$. First note that $W_{N}$ has rates $\left(2^{N-1} /\left(2^{N}-\right.\right.$ $\left.1), \cdots, 2^{N-i} /\left(2^{N}-1\right), \cdots, 1 /\left(2^{N}-1\right)\right)$. Then, we show that $W_{N}$ is balanced by induction. In the sequence $W_{N}$, any letter (say letter $j$ ) appears $2^{N-j}$ times in one period and is of the form of $2^{N-j}-1$ intervals of the same length $\left(2^{j}\right)$ and one of length $2^{j}-1$.

By construction of $W_{N+1}$, this properties still hold and therefore, $W_{N+1}$ is balanced.

Lemma 2.26. Let $W$ be balanced with rates $p_{1}>\cdots>p_{N}$, then, $W$ is periodic. In particular, this means that $p_{i} \in \mathbb{Q}, \forall 1 \leqslant i \leqslant N$.

Proof. If $W$ is not periodic, Theorem 2.17 says that $W$ is composed of two constant gap sequences. At least one of these sequences has at least two letters, and therefore two letters have rates which are equal by Lemma 2.12. Therefore, the rates in $W$ of these two letters are also equal.

Lemma 2.27. Let $W$ be balanced with rates $p_{1}>\cdots>p_{N}$, with the following property: for any $1 \leqslant i \leqslant N$, there exists two consecutive letters " $a_{i}$ " with no $a_{j}$ in between, with $j>i$. Then, $\left(p_{1}, \cdots, p_{N}\right)=\left(2^{N-1} /\left(2^{N}-1\right), \cdots, 2^{N-i} /\left(2^{N}-1\right), \cdots, 1 /\left(2^{N}-1\right)\right)$. 
This lemma is a partial "only if" result for the conjecture.

Proof. The proof holds by induction. Let $V_{k}$ denote the period of the balanced sequence with rates $\left(2^{k-1} /\left(2^{k}-1\right), \cdots, 2^{k-i} /\left(2^{k}-1\right), \cdots, 1 /\left(2^{k}-1\right)\right)$ given in Lemma 2.25. We recall that according to the construction in the proof of lemma $2.25, V_{k}=V_{k-1} a_{k} V_{k-1}$. We will prove by induction that $W$ is periodic with period $V_{N}$.

We first prove by induction on $k$ that $W$ contains the word $V_{k-1} a_{k} V_{k-1} V_{k-1} a_{k} V_{k-1}$ and that each letter in $W$, " $a_{j}$ ", $j \geqslant k$ is surrounded by $V_{k-1}$, for all possible $1<k \leqslant N$. Now, for the first step of the induction $(k=1)$, note that according to the property on $W, W$ contains the word " $a_{1} a_{1}$ " which is the same as " $V_{1} V_{1}$ ". Therefore any other letter is surrounded by two " $a_{1}$ "s. This also implies the existence of " $a_{1} a_{2} a_{1} a_{1} a_{2} a_{1}$ " by using a similar argument as step 2 in the proof of Theorem 2.20, which ends the case $k=1$.

For the general case, by the induction assumption, $a_{k}$ is surrounded by $V_{k-2}$, and we have the word " $V_{k-2} a_{k} V_{k-2}$ ". The existence of " $V_{k-2} a_{k-1} V_{k-2} V_{k-2} a_{k-1} V_{k-2}$ " proves that this word is surrounded with two " $a_{k-1}$ "s. Therefore, two consecutive $a_{k}$ form the word

$$
V_{k-2} a_{k-1} V_{k-2} a_{k} V_{k-2} a_{k-1} V_{k-2} U V_{k-2} a_{k-1} V_{k-2} a_{k} V_{k-2} a_{k-1} V_{k-2},
$$

where $U$ does not contain any letter " $a_{j}$ ", $j \geqslant k$. If $U$ contains " $a_{k-1}$ ", then $U$ is " $V_{k-2} a_{k-1} V_{k-2}$ ". This is impossible because of the existence of " $a_{k-1} V_{k-2} a_{k} V_{k-2} a_{k-1}$ ". If $U$ contains any other letter " $a_{i}$ ", then $U$ is reduced to this letter, and by construction of $V_{k-2}$, the presence of " $a_{i} V_{i-1} a_{i}$ " contradicts the existence of " $a_{i} V_{i-1} V_{i-1} a_{i}$ ". Therefore, $U$ is empty and we have the second part of the proof.

Now, we finish the proof by noticing that the letter $a_{N}$ is surrounded by $V_{N-1}$ and by noting that $V_{N-1}$ is necessarily surrounded by " $a_{N}$ ".

Lemma 2.28. Assume that $W$ is balanced. Assume that $p_{a} \geqslant 0.5$. Then the projection $W^{\prime}$ of $W$ over the alphabet $\mathcal{A}-\{a\}$ is also balanced.

Proof. Choose two words $V_{1}^{\prime}, V_{2}^{\prime}$ of length $n$ in $W^{\prime}$. Let $V_{1}$ and $V_{2}$ be any two words in $W$ whose projections over the alphabet $\mathcal{A}-\{a\}$ are $V_{1}^{\prime}$ and $V_{2}^{\prime}$, respectively. Assume, furthermore, that the first and last letters in $V_{1}$ and $V_{2}$ are not $a$. Let $k=\left|V_{1}\right|-n$ and $l=\left|V_{2}\right|-n$ denote the number of appearances of the letter $a$ in $V_{1}$ and $V_{2}$, resp.

Step 1: If $l=k$ then the difference in the number of occurrences of any letter $b$ in $V_{1}^{\prime}$ and in $V_{2}^{\prime}$ is at most 1 , since $W$ is most regular, and since the number of $b$ 's in $V_{1}$ (resp. $\left.V_{2}\right)$ is the same as its number in $V_{1}^{\prime}$ (resp. $\left.V_{2}^{\prime}\right)$.

Step 2: Assume that $l>k+1$.

- Let $\hat{V}_{2}$ be the word obtained from $V_{2}$ by truncating the first and last letter. Then $\left|\hat{V}_{2}\right|=n+l-2$, and the number of $a$ 's in $\hat{V}_{2}$ is $l$.

- Let $\hat{V}_{1}$ be the word obtained from $V_{1}$ by adding to it the next $m=l-k-2$ letters that appears after $V_{1}$ in the sequence $W$. Then $\left|\hat{V}_{1}\right|=n+l-2=\left|\hat{V}_{2}\right|$, and the number of $a$ 's in $\hat{V}_{1}$ is not larger than $k+m=l-2$. This is a contradiction with the fact that $W$ is balanced.

Step 3: It remains to check $l=k+1$. Add to $V_{1}$ the next letter that occurs in $W$ to its right, to form the new word $\bar{V}_{1}$. If it is not $a$ then we have two successive letters that are 
not $a$, which contradicts the fact that $a$ has an asymptotic frequency of at least $1 / 2$. If it is $a$, then $\bar{V}_{1}$ and $V_{2}$ have the same number of $a$ 's. We can now apply the same argument as in step 1 and conclude that the number number of occurrences of any letter $b$ in $V_{1}^{\prime}$ and in $V_{2}^{\prime}$ is at most 1 .

Combining the above steps, we conclude that $W^{\prime}$ is balanced.

\subsection{Extensions of the original problem}

So far we have only analyzed the case where all the rates add up to one. The different results tend to prove that very few rates are balanced.

Now let us look at a generalization when all the rates do not add up to one. Assume that $S$ is a sequence on the alphabet $\left\{a_{1}, a_{2}, \cdots, a_{k}, *\right\}$. We only require that $S$ is balanced for the letters $a_{1}, \cdots, a_{k}$, but not for the special letter $*$.

On a more practical point of view, the question can be viewed as whether this allows more possibilities for rates to be balanced when "losses" are allowed (represented by the letter $*$ ). Then again, in general, the rates are not balanced, even if the total sum is very small as illustrated by the following lemma.

Lemma 2.29. For an arbitrary $\varepsilon>0$, there exists two real numbers $p_{1}$ and $p_{2}$ such that $p_{1}+p_{2}<\varepsilon$ and no sequence $S$ on the alphabet $\{a, b, *\}$ with asymptotic rate $p_{1}$ for letter a and $p_{2}$ for letter $b$ which is balanced for $a$ and $b$.

Proof. Choose two irrational numbers $p_{1}$ and $p_{2}$ with $p_{1}+p_{2}<\varepsilon$ such that $p_{1}, p_{2}$ and 1 are not linearly dependent on $\mathbb{Z}$. Now assume that there exists a sequence $S$ on $\{a, b, *\}$ with asymptotic rate $p_{1}$ for letter $a$ and $p_{2}$ for letter $b$ which is balanced for $a$ and $b$. By Theorem 2.5, then there exists two real numbers $x, y$ such that $\delta_{a}(S)(n)=1$ if $x+p_{1} n$ $\bmod 1 \in\left[1-p_{1}, 1\right]$ and 0 otherwise. $\delta_{b}(S)(n)=1$ if $y+p_{2} n \bmod 1 \in\left[1-p_{2}, 1\right]$ and 0 otherwise. In the cube $[0,1]^{2}$, the set of points $(x, y)+n\left(p_{1}, p_{2}\right) \bmod (1,1)$ is dense (see for example, Weyl's ergodic theorem [19]) and therefore hits the rectangle $\left[\left(1-p_{1}, 1-p_{2}\right),(1,1)\right]$. This is not possible.

More on this kind of problems can be found in [21].

To end this short overview on balanced sequences, we must mention on the positive side that "usual" rates, such as $(1 / k, 1 / k, \cdots, 1 / k)$ are often balanceable. In Appendix 4.3, some examples of balanced sequences and their rates are given.

\section{Routing of customers in multiple queues}

The notion of balanced sequences gives birth to an large set of elegant properties in word combinatorics. However, they are rarely used in other domains. The balanced sequences in dimension $N=2$ have been used for discrete line drawing [14] and also for scheduling optimization in $[10,8]$.

However, the case $N=2$ behaves differently than for higher dimension (as illustrated by theorem 2.18) and does not really grasp all the complexity of the model. 
Here, we present an application of balanced sequences in arbitrary dimensions to scheduling optimization.

We consider a system where a sequence of tasks have to be executed by several processing units. The tasks arrive sequentially and each task can be processed by any server. The routing control consists in assigning to each task a server on which it will be processed. The routing is optimal it it minimizes some cost function that measures the performance of the system.

These kinds of models have used to study load balancing within several processors in parallel processing problems as well as for efficient network utilization in telecommunication systems.

\subsection{Presentation of the model}

In this section we consider a more precise queueing model of the system that we described. Customers enter a multiple queue system composed of $K$ nodes. Each node is made of several queues which form an event graph. Event graphs are a subset of Petri nets with no more than one input transition and one output transition per place. More details on the dynamics of event graphs can be found in [4]. In particular, their dynamical behavior is linear in the so-called (max,+) algebra ([4]). Many queueing networks can be represented as event graphs, as long as there is no inside choice for the route followed by the customers. (see [1] for a more complete discussion on this issue).

The routing of customers to the different nodes is controled by a sequence of vectors $\left\{a_{n}\right\}$, with $a_{n}$ is in $\{0,1\}^{K}$ and $a_{n}^{i}=1$ means that the $n$-th customer is routed to queue $i$. Note that $a$ is a feasible admission sequence as long as for all $n, \sum_{i} a_{n}^{i}=1$.

The link between a feasible routing policy and an infinite sequence on a finite alphabet comes from choosing the alphabet $\mathcal{A}$ composed by the letters

$$
\{(1,0, \cdots, 0),(0,1,0, \cdots), \cdots,(0, \cdots, 0,1)\} .
$$

Using this alphabet on $K$ letters, a feasible routing policy can be viewed as an infinite sequence on $\mathcal{A}$.

Figure 1 shows an illustration of the system we are considering.

We denote by $T_{n}$ the epoch when the $n$-th customer enters the system. We assume that

$T_{1}=0$. The inter-arrival time sequence is $\left\{\delta_{k}\right\} \stackrel{\text { def }}{=}\left\{T_{i+1}-T_{i}\right\}$. Finally, $\sigma_{n}^{i, j}$ will denote the service time of the $n$-th customer entering the $j$-th queue in node $i$.

The sequences $\left\{\delta_{k}\right\}$ and $\left\{\sigma_{k}^{i, j}\right\}$ will be considered as random processes. We also make stochastic assumption on these sequences. The inter-arrival time of the customers and the service times form stationary processes, and we assume that the inter-arrival times are independent of the service times.

\subsection{Optimal admission sequence}

In each node $i$ we pick an arbitrary server $s_{i}$ (which may be the last server in the node for example). The performance criteria for node $i$ will be the traveling time to server $s_{i}$ 


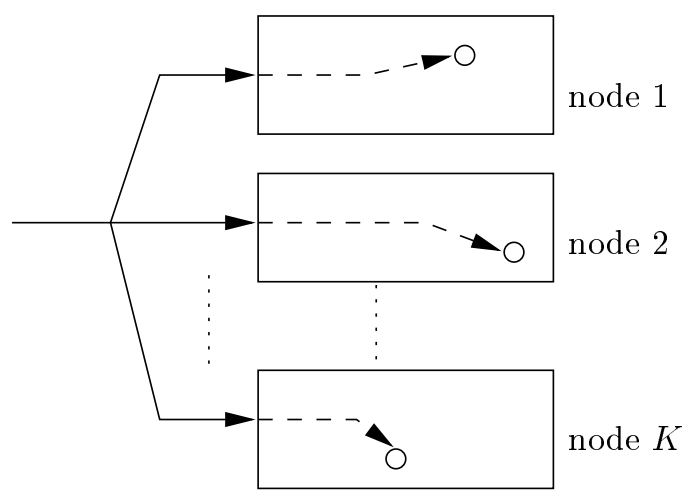

Figure 1: Illustration of the routing of customers in a $K$ node system

of a virtual customer that would enter node $i$ at time $T_{n}$. Under the routing policy $a$, this quantity only depends on the values of the $n$ first routing choices. From the routing sequence $a$, we can isolate the routing decision for node $i$ : if $a_{n}^{i}=1$ then the customer is admitted in node $i$ and if $a_{n}^{i}=0$ then the customer is rejected (for node $i$ ). We denote the traveling time at time $T_{n}$ by $W_{n}^{i}\left(a_{1}^{i}, \cdots, a_{n}^{i}\right)$. We will be more particularly interested in the expected value of the traveling time with respect to the service times in all the servers contained in node $i$ and with respect to the inter-arrival times: $\mathbf{W}_{n}^{i}\left(a_{1}^{i}, \cdots, a_{n}^{i}\right) \stackrel{\text { def }}{=}$ $\mathbf{E}_{\sigma, \tau} f\left(W_{n}^{i}\left(a_{1}^{i}, \cdots, a_{n}^{i}\right)\right)$, where $f: \mathbb{R} \rightarrow \mathbb{R}$ is any convex increasing function.

If we focus on a single node $i$, the function $\mathbf{W}_{n}^{i}\left(a_{1}^{i}, \cdots, a_{n}^{i}\right)$ has been studied in [1]. Its most remarkable property is the fact that this function is multimodular. See [10,2] for a precise definition and several properties of multimodular functions. Here, we merely point out that multimodularity is closely to convexity (see [2]).

Proposition 3.1 ([1]). Under the foregoing assumptions, the function $\mathbf{W}_{n}^{i}\left(a_{1}^{i}, \cdots, a_{n}^{i}\right)$ satisfies the following properties.

(1)- $\mathbf{W}_{n}^{i}\left(a_{1}^{i}, \cdots, a_{n}^{i}\right)$ is increasing in $a_{k}^{i}, 1 \leqslant k \leqslant n$,

(2)- $\mathbf{W}_{m}^{i}\left(0, \cdots, 0, a_{1}^{i}, \cdots, a_{n}^{i}\right)=\mathbf{W}_{n}^{i}\left(a_{1}^{i}, \cdots, a_{n}^{i}\right)$, if $m>n$,

(3)- $\mathbf{W}_{n}^{i}\left(a_{1}^{i}, \cdots, a_{n}^{i}\right)$ is multimodular.

Proof. These properties are shown in [1].

Using these properties, one can derive as in [2], a lower bound $B_{i}(\alpha, p)$ (which is increasing in $\alpha$ and $p$ and continuous) for any routing $a^{i}$, for the following discounted cost.

$$
\sum_{n=1}^{\infty}(1-\alpha) \alpha^{n-1} \mathbf{W}_{n}^{i}\left(a_{1}^{i}, a_{2}^{i} \ldots, a_{n}^{i}\right) \geqslant B_{i}\left(\alpha, p_{\alpha}\right),
$$

where $p_{\alpha} \stackrel{\text { def }}{=}(1-\alpha) \sum_{k=1}^{\infty} \alpha^{k-1} a_{k}^{i}$.

Also, for a given $p$, we define the regular sequence with rate $p$ and arbitrary phase $\theta$,

$$
a^{p}(\theta) \stackrel{\text { def }}{=}\lfloor n p+\theta\rfloor-\lfloor(n-1) p+\theta\rfloor,
$$


(see the definition 2.4). One can show as in [2] that $a^{p}(\theta)$ satisfies

$$
\lim _{m \rightarrow \infty} \frac{1}{m} \sum_{n=1}^{m} \mathbf{W}_{n}^{i}\left(a_{1}^{p}(\theta), \cdots, a_{n}^{p}(\theta)\right)=B_{i}(1, p) .
$$

Here, however, we are interested in the performance of all nodes together. Therefore, we choose as a cost function, the undiscounted average on $n$ of some linear combination of the expected traveling time in all nodes.

Let $h$ be any increasing linear function, $h: \mathbb{R}^{K} \rightarrow \mathbb{R}$. We consider the undiscounted average cost of a feasible routing sequence $a$,

$$
g(a) \stackrel{\text { def }}{=} \varlimsup_{\lim _{N \rightarrow \infty}} \frac{1}{N} \sum_{n=1}^{N} h\left(\mathbf{W}_{n}^{1}, \ldots, \mathbf{W}_{n}^{K}\right) .
$$

From this point on, we mimic the general method developed in [2] for our case.

We use the following notation.

$$
B_{i}\left(p_{i}\right) \stackrel{\text { def }}{=} \sup _{\alpha \leqslant 1} B_{i}\left(\alpha, p_{i}\right)
$$

Our objective is to minimize $g(a)$.

Theorem 3.2. The following lower bound holds for all policies:

$$
g(a) \geqslant \inf _{p_{1}+\ldots+p_{K}=1} h\left(B_{1}\left(p_{1}\right), \ldots, B_{K}\left(p_{K}\right)\right) .
$$

Proof. Due to Littlewood's and Jensen's inequalities as well as Equation 5, we have

$$
\begin{aligned}
\varlimsup_{N \rightarrow \infty} & \frac{1}{N} \sum_{n=1}^{N} h\left(\mathbf{W}_{n}^{1}, \ldots, \mathbf{W}_{n}^{K}\right) \\
\geqslant & \varlimsup_{\alpha \rightarrow 1}(1-\alpha) \sum_{n=1}^{\infty} \alpha^{n-1} h\left(\mathbf{W}_{n}^{1}, \ldots, \mathbf{W}_{n}^{K}\right) \\
\geqslant & \varlimsup_{\alpha \rightarrow 1} h\left((1-\alpha) \sum_{n=1}^{\infty} \alpha^{n-1} \mathbf{W}_{n}^{1}, \ldots,(1-\alpha) \sum_{n=1}^{\infty} \alpha^{n-1} \mathbf{W}_{n}^{K}\right) \\
\geqslant & \varlimsup_{\alpha \rightarrow 1} h\left(B_{1}\left(\alpha, p_{1}^{a}(\alpha)\right), \ldots, B_{K}\left(\alpha, p_{K}^{a}(\alpha)\right)\right)
\end{aligned}
$$

where

$$
p_{i}^{a}(\alpha) \stackrel{\text { def }}{=}(1-\alpha) \sum_{k=1}^{\infty} \alpha^{k-1} a_{k}^{i}
$$


We note that $\sum_{i=1}^{K} p_{i}^{a}(\alpha)=1$. Hence, one may choose a sequence $\alpha_{n} \uparrow 1$ such that the following limits exist:

$$
\lim _{n \rightarrow \infty} p_{i}^{a}\left(\alpha_{n}\right)=p_{i}, \quad i=1, \ldots, K
$$

and $\sum_{i=1}^{K} p_{i}=1$. From the continuity of $B_{i}\left(\alpha, p_{i}\right)$ in $p$ and $\alpha$ we get from $(8)$

$$
\begin{aligned}
g(a) & \geqslant h\left(B_{1}\left(p_{1}\right), \ldots,, B_{K}\left(p_{K}\right)\right) \\
& \geqslant \inf _{p_{1}+\ldots+p_{K}=1} h\left(B_{1}\left(p_{1}\right), \ldots, B_{K}\left(p_{K}\right)\right) .
\end{aligned}
$$

Note that there exists some $p^{*}$ that achieves the infimum

$$
\inf _{p_{1}+\ldots+p_{K}=1} h\left(B_{1}\left(p_{1}\right), \ldots, B_{K}\left(p_{K}\right)\right)
$$

since $h\left(B_{1}\left(p_{1}\right), \ldots, B_{K}\left(p_{K}\right)\right)$ is continuous in $p=\left(p_{1}, \cdots, p_{K}\right)$.

Consider $\theta=\left(\theta_{1}, \cdots, \theta_{K}\right)$ and the routing policy $a^{p^{*}}(\theta)$ given for each $i$ by

$$
a_{k}^{i, p^{*}}\left(\theta_{i}\right)=\left\lfloor k p_{i}^{*}+\theta_{i}\right\rfloor-\left\lfloor(k-1) p_{i}^{*}+\theta_{i}\right\rfloor .
$$

There are some $p^{*}$ 's for which the condition of feasibility of the policy $a^{p^{*}}(\theta)$ is satisfied, that is, there exists some $\theta=\left(\theta_{1}, \ldots, \theta_{K}\right)$, such that the regular policy $a^{p^{*}}(\theta)$ is feasible.

Using the correspondence between a routing policy and a sequence on the alphabet $\mathcal{A}$, these $p^{*}$ 's correspond precisely to balanceable rates.

Theorem 3.3. Assume that $p^{*}$ is balanceable. Then $a^{p^{*}}(\theta)$ is optimal for the average cost, i.e. it minimizes $g(a)$ over all feasible policies.

Proof. The proof follows directly from Theorem 3.2 and Equation (6).

\section{Study of some special cases}

The problem which remains to be addressed is to find in which cases, the rate vector $p^{*}$ is balanceable. We will present several simple examples for which we can make sure that the optimal rate $p^{*}$ is balanceable.

\subsection{The case $K=2$}

If $K=2$, then, the optimal rate vector is of the form $p^{*}=\left(p_{1}^{*}, 1-p_{1}^{*}\right)$. Theorem 2.18 says that $p^{*}$ is always balanceable and therefore, the optimal routing sequence is given by an associated balanced sequence. Note that this approach does not give any direct way to compute the value of $p^{*}$, however, it gives the structure of the optimal policy. 


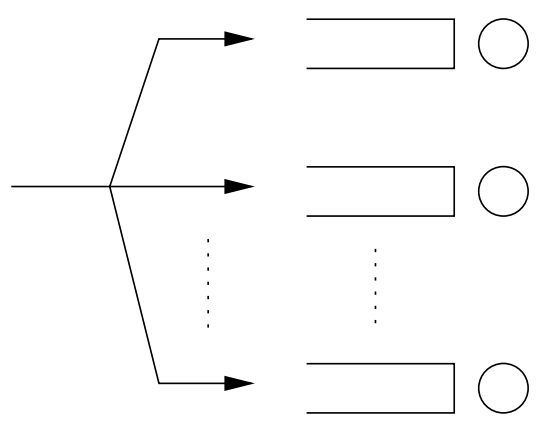

Figure 2: Routing in homogeneous queues.

\subsection{The homogeneous case}

Now let $K$ be arbitrary and each node is made of a single server, all servers being identical. This model is displayed in Figure 2.

Also assume that the function $h$ is symmetrical in all coordinates (for example, just the sum of all waiting times) By symmetry and convexity in $\left(p_{1}, \cdots, p_{K}\right)$ of the function $h\left(V_{1}^{1}\left(p_{1}\right), \ldots, V_{K}^{1}\left(p_{K}\right)\right)$, then, $p^{*}=(1 / K, \cdots, 1 / K)$, which is balanceable. The associated balanced sequence is the round robin routing scheme. Applying Theorem 3.3 yields the following result which is new (to the best of the author's knowledge).

Theorem 4.1. The round robin routing to $K$ identical ./G/1 queues, minimizes the total average expected workload of all the queues over all admission sequences with no information on the state of the system.

In [12], the round robin routing is proved to be optimal in separable-convex increasing order for $K$ identical ./GI/1 queues. Their method uses an intricate coupling argument, whereas our proof is a simple corollary of the general theory on multimodular functions.

To illustrate the advantage of our approach, we further generalize the result to a system composed of $K$ identical ( $\max ,+$ ) linear systems with a single entry. In this case, the symmetry argument used in the case of simple queues still holds. Then again, the round robin routing policy minimizes the traveling time in each system. This case includes models such as routing among several identical systems composed of queues in tandem, for example (see Figure 3).

\subsection{Two sets of identical servers}

As a consequence of the two previous cases, we can consider a system composed of $K_{1}$ identical queues of type 1 and $K_{2}$ queues of type 2. Again, assume that $h$ is symmetrical in the $K_{1}$ nodes of type 1 and symmetrical in the $K-2$ nodes of type 2 . Then, by symmetry arguments, the optimal rate vector $p^{*}$ is of the form

$$
\left(\frac{p}{K_{1}}, \cdots, \frac{p}{K_{1}}, \frac{1-p}{K_{2}}, \cdots, \frac{1-p}{K_{2}}\right) .
$$




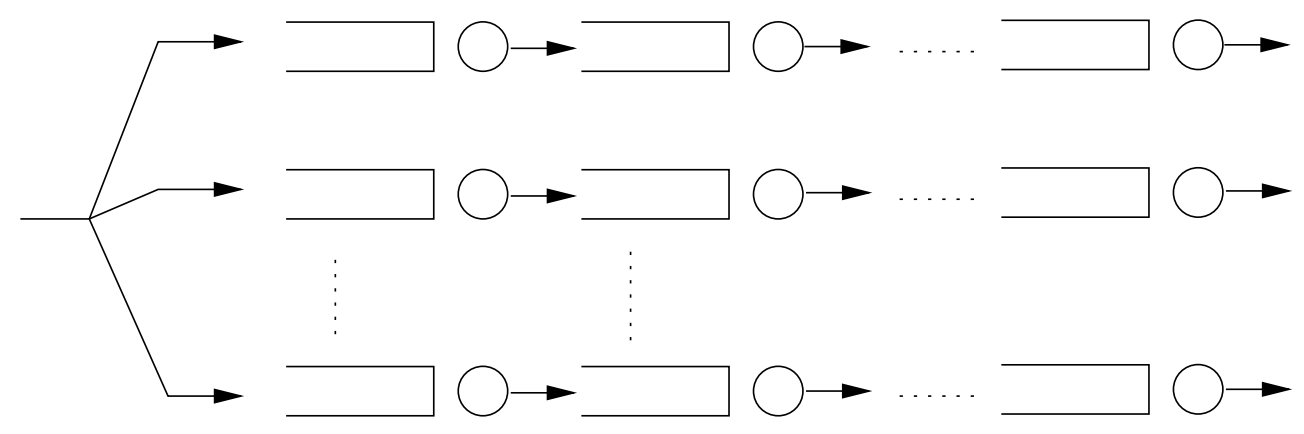

Figure 3: Routing in queues in tandem

This rate vector is balanceable indeed. This implies that for the weighted total average expected workload, the optimal routing is of balanced type, if nodes of the same type have the same weight.

Many other examples of this kind can be derived from these examples through similar constructions.

\section{Appendix}

Here is a collection of balanceable rates. We also give a corresponding balanced sequence.

- $(1 / 7,2 / 7,4 / 7)$ is balanceable and $S=(\text { abacaba })^{\omega}$.

- $(1 / 11,2 / 11,4 / 11,4 / 11)$ is balanceable and $S=(\text { abcababcabd })^{\omega}$.

- $(1 / 11,2 / 11,2 / 11,6 / 11)$ is balanceable and $S=(\text { abacaabacad })^{\omega}$.

- $(1 / 11,1 / 11,3 / 11,6 / 11)$ is balanceable and $S=\left(\right.$ acabaabadab $^{\omega}$.

- $(1 / 14,1 / 14,4 / 14,8 / 14)$ is balanceable and $S=(\text { abacabaabadaba })^{\omega}$.

- For all real number $0<p \leqslant 1$, the rates $(1-p, p / 4, p / 4, p / 2)$ are balanceable, with a corresponding balanced sequence constructed from a regular sequence with rate $p$ where all 1 are replaced in turn by the sequence $(a b a c)^{\omega}$ and each 0 by the letter $d$.

- for all $N,\left(\left(2^{N-1} /\left(2^{N}-1\right), \cdots, 2^{N-i} /\left(2^{N}-1\right), \cdots, 1 /\left(2^{N}-1\right)\right)\right)$ is balanceable. The associated balanced sequence is constructed recursively as in Lemma 2.25.

- $(1 / k, \cdots, 1 / k)$ is balanceable. A balanced sequence is: $S=\left(a_{1} a_{2} a_{3} \cdots a_{k}\right)^{\omega}$.

- $(p, \cdots, p, \beta, \cdots, \beta)$ is balanceable. A balanced sequence with those rates is constructed in the following way: Choose a balanced sequence $S$ on letters, $(A, B)$ with rate $\left(\sum_{i=1}^{k} p, \sum_{i=1}^{h} \beta\right.$ ). In $S$ replace all the $A$ (resp. $B$ ) by $a_{1}, a_{2}, \cdots, a_{k}$ (resp. $\left.b_{1}, \cdots, b_{h}\right)$ in a round robin fashion to get a balanced sequence with the required rates. 


\section{Acknowledgment}

The authors would like to thank Alain Jean-Marie who pointed out the reference [23] and gave them a first proof of Lemma 2.12 .

\section{References}

[1] E. Altman, B. Gaujal, and A. Hordijk. Admission control in stochastic event graphs. Technical Report 3179, INRIA, June 1997.

[2] E. Altman, B. Gaujal, and A. Hordijk. Multimodularity, convexity and optimization properties. Technical Report 3181, INRIA, June 1997.

[3] P. Arnoux, C. Mauduit, I. Shiokawa, and J. Tamura. Complexity of sequences defined by billiards in the cube. BSMF (Bulletin de la Societé Mathématique de France), 122:1-12, 1994.

[4] F. Baccelli, G. Gohen, G.J. Olsder, and J.-P. Quadrat. Synchronization and Linearity. Wiley, 1992.

[5] Y. Baryshnikov. Complexity of trajectories in rectangular billiards. Com. Math. Phys., 175:43-56, 1995.

[6] J. Berstel and P. Seébold. Morphismes de Sturm. Bull. Belg. Math. Soc., 1:175-189, 1994.

[7] A. Fraenkel. Complementary and exactly covering sequences. Journal of Combinatorial Theory, 14:8-20, 1973.

[8] B. Gaujal. Optimal allocation sequences of two processes sharing a resource. Discrete Event Dynamic Systems, 1997. to appear.

[9] R. Graham. Covering the positive integers by disjoint sets of the form $\{[n \alpha+\beta]: n=$ 1,2, ․ . J. Combi. Theory, 15:354-358, 1973.

[10] B. Hajek. Extremal splittings of point processes. Mathematics of Operation Research, 10(4):543-556, 1985.

[11] P. Hubert. Suites équilibrées. Technical report, Université de Marseille, 1996.

[12] Z. Liu and R. Righter. Optimal load balancing on distrinuted homogeneous unreliable processors. Journal of Operations Research, 1997. To appear.

[13] J. A. Loeve. Markov Desicion Chains With Partial Information. PhD thesis, Leiden University, 1995. Chap 8: Regularity of words and periodic policies. 
[14] M. Lothaire. Mots, chapter Tracé de droites, fractions continues et morphismes itérés (J. Berstel). Hermes, 1991.

[15] R. Morikawa. Disjoint sequences generated by the bracket function i-vi. Technical Report 26(1985), 28(1988), 30(1989), 32(1992), 34(1993), Bull. Faculty of Liberal Arts, Nagasaki Univ., 1993.

[16] R. Morikawa. On eventually covering families generated by the bracket function i-v. Technical Report 23(1982), 24(1983), 25(1984), 26(1985), 25(1985), 36(1995), Bull. Faculty of Liberal Arts, Nagasaki Univ., 1995.

[17] M. Morse and G. A. Hedlund. Symbolic dynamics II- Sturmian trajectories. American Journal Math., 62:287-306, 1940.

[18] M. Newman. Roots of unity and covering sets. Math. Ann., 191:279-282, 1971.

[19] Y. G. Sinai. Introduction to Ergodic Theory. Princeton University Press, Princeton, N.J., 1976.

[20] R. Tijdeman. On complementary triples of sturmian sequences. Technical Report W95-13, Leiden University, The Netherlands, 1995.

[21] R. Tijdeman. On disjoint pairs of sturmian bisequences. Technical report, Leiden University, The Netherlands, 1995.

[22] L. Vuillon. Combinatoire des motifs d'une suite sturmienne bidimensionnelle. Theoritical Computer Science, 1997. To appear.

[23] H. S. Wilf. Generatingfunctionology. Academic Press, 2nd edition, 1994. 